\title{
GENE LINKAGE TEST FOR imr WITH ol, tph1 AND tph2 MUTATIONS IN SUNFLOWER
}

\author{
Demurin, Ya.N. ${ }^{*}$, Borisenko, O.M., Peretyagina, T.M., Perstenyeva, A.A.
}

All-Russia Research Institute of Oil Crops (VNIIMK), 350038 Filatova 17, Krasnodar, Russia

Received: September 10, 2005 Accepted: March 25, 2006

\section{SUMMARY}

The objective of this research was to check possibilities of combining imidazolinone resistance with mutations for seed oil quality characters in sunflower. An imidazolinone resistant line HA425 (ImrImr) with normal fatty acid and tocopherol compositions was used as female after hand emasculation. An imidazolinone susceptible inbred line VK876 with high oleic content and changed tocopherol composition (OlOl tph1tph1 tph2tph2) served as male. Linkage test in the $F_{2}$ and $F_{3}$ generations has shown the Imr gene for imidazolinone resistance to be independently inherited from the high oleic mutation $\mathrm{Ol}$, high $\beta$-tocopherol mutation tph1 and high $\gamma$-tocopherol mutation tph2. Recombinant genotypes have been selected for development of inbred lines.

Key words: imidazolinone resistance, inheritance, oleic mutation, tocopherols

\section{INTRODUCTION}

Resistance to imidazolinone herbicides was found in a population of wild sunflower (Al-Khatib et al., 1998). This character was shown to be controlled by one semi-dominant gene Imr1 and a modifier gene Imr2 (Bruniard and Miller, 2001).

A high oleic genotype Pervenets was developed as a result of DMS chemical mutagenesis on the seeds of the variety VNIIMK 8931 (Soldatov, 1976). The first research on genetic control of the high oleic mutation led to the hypothesis of single dominant gene, $\mathrm{Ol}$ (Fick, 1984).

Two non-allelic unlinked genes, Tph1 and Tph2, which control tocopherol composition in sunflower seeds were identified (Demurin, 1993). Recessive alleles of the genes were found by wide-scale screening and selfing as spontaneous mutations. Tph 1 gene controls the ratio of $\alpha$ - and $\beta$-tocopherols, whereas Tph 2 gene affects that of $\alpha$ - and $\gamma$-homologues. The tph 2 mutation has an epistatic action over tph 1 with $\delta$-tocopherol appearance.

* Corresponding author: e-mail: yakdemurin@yandex.ru 
The objective of this research was to test the linkage of the above genes. The answer will provide breeders with information of possibility to combine desired characters in one phenotype.

\section{MATERIALS AND METHODS}

HA425, an imidazolinone resistant inbred line with normal fatty acid and tocopherol composition was used as female after hand emasculation. VK876, an imidazolinone susceptible inbred line with high oleic content and changed tocopherol compositin (OlOl tph1tph1 tph2tph2) served as male.

Crosses were made at the experiment field of VNIIMK, Krasnodar, in the summer of 2003. $F_{1}$ plants were grown and self-pollinated in the greenhouse in the winter of 2003-2004. $\mathrm{F}_{2}$ plants were grown and self-pollinated in the field in the summer of $2004 . \mathrm{F}_{3}$ populations from $\mathrm{F}_{2}$-derived plants were tested in the field in the summer of 2005.

$\mathrm{P}, \mathrm{F}_{1}, \mathrm{~F}_{2}$ and $\mathrm{F}_{3}$ plants were treated at the V6 stage with imazethapyr (Pivot) at a $7 \times$ rate $\left(6 \mathrm{ml} \mathrm{l}^{-1}\right.$ or $245 \mathrm{~g} \mathrm{AI} \mathrm{ha}^{-1}$ or $4 \mathrm{mg} \mathrm{AI}$ per a plant).

Fatty acid composition in seeds bulk was determined by gas chromatography of methyl esters.

Tocopherol composition was estimated with thin-layer chromatography (TLC) followed by Emmerie-Engel reaction without corrections of the different rates of homologue staining (Popov et al., 1991).

\section{RESULTS AND DISCUSSION}

The parent lines HA425 and VK876 confirmed to be resistant and susceptible (died after seven days) to imidazolinone herbicide, respectively. $\mathrm{F}_{1}$ intermediate plants showed a definite degree of chlorosis symptoms. After combining the number of resistant and intermediate plants as survived in the $F_{2}$ and $F_{3}$ segregating populations, one semi-dominant Imr gene model was accepted (Table 1). Moreover, the five homogeneous and seven $\mathrm{F}_{3}$ populations segregating for resistance fit an expected ratio of $1: 2\left(\chi_{1: 1}^{2}=0.34<\chi^{2}{ }_{05}=3.84\right)$. That was due to the lack of susceptible $\mathrm{F}_{3}$ populations because susceptible plants had died after herbicide treatment in the $\mathrm{F}_{2}$ generation. Thus the recessive homozygote imr imr was eliminated in $\mathrm{F}_{2}$.

Two out of twelve $\mathrm{F}_{3}$ populations, numbered 1 and 4 , were high oleic phenotypes with more than $82 \%$ of oleic acid. Two other populations, numbered 6 and 9 , were clearly low oleic (Table 2). The ratio of 2 homogeneous high oleic: 10 segregated: 2 homogeneous low oleic fit the expected ratio $1: 2: 1$ with $\chi_{1: 2: 1}^{2}=3.33<$ $\chi^{2} 05=5.99$. 
Table 1: Inheritance of imidazolinone resistance in sunflower

\begin{tabular}{|c|c|c|c|c|}
\hline \multirow{2}{*}{\multicolumn{2}{|c|}{$\begin{array}{l}\text { Generation, } \\
\text { population }\end{array}$}} & \multicolumn{2}{|c|}{ Plant number } & \multirow{2}{*}{$\left.\chi_{05}^{\chi^{2} 3: 1}=3.84\right)$} \\
\hline & & Resistant & Susceptible & \\
\hline \multicolumn{2}{|c|}{ HA425 우 } & 20 & 0 & - \\
\hline \multicolumn{2}{|c|}{ HA876 ô } & 0 & 14 & - \\
\hline \multicolumn{2}{|l|}{$\mathrm{F}_{1}$} & 19 & 0 & - \\
\hline \multicolumn{2}{|l|}{$\mathrm{F}_{2}$} & 98 & 32 & 0.01 \\
\hline \multirow[t]{12}{*}{$\mathrm{F}_{3}$} & 1 & 24 & 0 & - \\
\hline & 2 & 16 & 6 & 0.06 \\
\hline & 3 & 23 & 0 & - \\
\hline & 4 & 27 & 0 & - \\
\hline & 5 & 19 & 4 & 0.40 \\
\hline & 6 & 22 & 0 & - \\
\hline & 7 & 20 & 3 & 1.75 \\
\hline & 8 & 12 & 3 & 0.20 \\
\hline & 9 & 19 & 6 & 0.01 \\
\hline & 10 & 20 & 5 & 0.33 \\
\hline & 11 & 21 & 5 & 0.46 \\
\hline & 12 & 20 & 0 & - \\
\hline
\end{tabular}

Table 2: Fatty acid composition in seed bulk of imidazolinone resistant $\mathrm{F}_{2}$-derived $\mathrm{F}_{3}$ populations

\begin{tabular}{lcccc}
\hline $\begin{array}{l}\text { Population } \\
\text { index }\end{array}$ & \multicolumn{4}{c}{ Fatty acid composition, \% } \\
\cline { 2 - 5 } 1 & Palmitic & Stearic & Oleic & Linoleic \\
\hline 2 & 7 & 2 & 84 & 7 \\
3 & 8 & 5 & 31 & 56 \\
4 & 7 & 3 & 46 & 10 \\
5 & 5 & 3 & 82 & 56 \\
6 & 7 & 3 & 34 & 64 \\
7 & 8 & 3 & 25 & 53 \\
8 & 7 & 5 & 35 & 38 \\
9 & 8 & 6 & 48 & 68 \\
10 & 8 & 6 & 18 & 51 \\
11 & 8 & 7 & 34 & 49 \\
12 & 8 & 6 & 37 & 49 \\
\hline
\end{tabular}

Theoretically, nine types of inheritance for two tocopherol genes can be observed in $\mathrm{F}_{3}$ seeds at the ratio $4(9 \alpha: 3 \beta: 3 \gamma: 1 \delta): 2(3 \alpha: 1 \beta): 2(3 \alpha: 1 \gamma): 2(3 \beta: 1 \delta): 2$ $(3 \gamma: 1 \delta): 1($ all $\alpha): 1$ (all $\beta$ ) : 1 (all $\gamma): 1$ (all $\delta$ ) when $\alpha, \beta, \gamma$ and $\delta$ correspond to normal, tph1, tph2 and double mutation phenotypes. Six types were found with exception of 3a: $1 \beta$, all $\alpha$ and all $\delta$. Three populations were homogeneous in tocopherol composition, 3 and 7 with $\beta$-form and 10 with $\gamma$-form (Table 3 ). 
Table 3: Segregation for tocopherol genes in individual seeds of the $F_{3}$ populations

\begin{tabular}{lcccc}
\hline \multirow{2}{*}{$\begin{array}{l}\text { Population } \\
\text { index }\end{array}$} & $\begin{array}{c}\text { Tph1- } \\
\text { Tph2- }\end{array}$ & $\begin{array}{c}\text { tph1tph1 } \\
\text { Tph2- }\end{array}$ & $\begin{array}{c}\text { Tph1- } \\
\text { tph2tph2 }\end{array}$ & $\begin{array}{c}\text { tph1tph1 } \\
\text { tph2tph2 }\end{array}$ \\
\hline 1 & 0 & 0 & 15 & 4 \\
2 & 5 & 2 & 8 & 1 \\
3 & 0 & 19 & 0 & 0 \\
4 & 0 & 15 & 0 & 4 \\
5 & 9 & 2 & 5 & 2 \\
6 & 15 & 0 & 4 & 0 \\
7 & 0 & 19 & 0 & 0 \\
8 & 0 & 0 & 14 & 5 \\
9 & 1 & 11 & 4 & 3 \\
10 & 0 & 0 & 38 & 0 \\
11 & 0 & 12 & 0 & 6 \\
12 & 2 & 1 & 14 & 2 \\
\hline
\end{tabular}

Recombinant homozygous genotypes in $\mathrm{F}_{2}$ ImrImr and OlOl (plant 1, 4), ImrImr and tph1tph1 (3, 4), ImrImr and tph2tph2 (1) were obtained (Table 4). It means that imidazolinone resistance was combined in one phenotype with high oleic acid content, high $\beta$-tocopherol content or high $\gamma$-tocopherol content.

Table 4: Deduced $\mathrm{F}_{2}$ genotypes after estimation of $\mathrm{F}_{3}$ populations

\begin{tabular}{lcccc}
\hline Plant & & \multicolumn{2}{c}{ Character } \\
\hline 1 & Imi-resistance & Oleic content & $\beta$-tocopherol & $\gamma$-tocopherol \\
\cline { 2 - 5 } 2 & Imrlmr & OIOI & Tph1tph1 & tph2tph2 \\
3 & Imrimr & Olol & Tph1tph1 & Tph2tph2 \\
4 & Imrlmr & Olol & tph1tph1 & Tph2Tph2 \\
5 & Imrlmr & OlOI & tph1tph1 & Tph2tph2 \\
6 & Imrimr & Olol & Tph1tph1 & Tph2tph2 \\
7 & Imrlmr & olol & Tph1Tph1 & Tph2tph2 \\
8 & Imrimr & Olol & tph1tph1 & Tph2Tph2 \\
9 & Imrimr & Olol & Tph1tph1 & tph2tph2 \\
10 & Imrimr & olol & Tph1tph1 & Tph2tph2 \\
11 & Imrimr & Olol & Tph1Tph1 & tph2tph2 \\
12 & Imrimr & Olol & tph1tph1 & Tph2tph2 \\
\hline
\end{tabular}

\section{CONCLUSIONS}

The linkage test in the $\mathrm{F}_{2}$ and $\mathrm{F}_{3}$ generations showed the Imr gene for imidazolinone resistance to be independently inherited from the high oleic mutation $\mathrm{Ol}$, high $\beta$-tocopherol mutation tph1 and high $\gamma$-tocopherol mutation tph2. Recombinant genotypes were selected for development of inbred lines. 


\title{
ACKNOWLEDGEMENTS
}

The authors wish to thank Dr. J.F. Miller from USDA-ARS, Northern Crop Science Laboratory, Fargo, ND, USA, for the seeds of sunflower line HA425.

Support from RFFI, Russia, grant no. 03-04-96759, is gratefully acknowledged.

\section{REFERENCES}

Al-Khatib, K., Baumgartner, J.R., Peterson, D.E. and Currie, R.S., 1998. Imazethapyr resistance in common sunflower (Helianthus annuus). Weed Sci., 46: 403-407.

Bruniard, J.M. and Miller, J.F., 2001. Inheritance of imidazolinone-herbicide resistance in sunflower. Helia 24(35): 11-16.

Demurin, Ya., N., 1993. Genetic variability of tocopherol composition in sunflower seeds. Helia 16(18): 59-62

Fick, G.N., 1984. Inheritance of high oleic acid in the seed oil of sunflower. In: Proc. Sunflower Res. Workshop, National Sunflower Association, Bismarck, ND: 9.

Popov, P.S. and Aspiotis, E.H., 1991. Biochemical methods for breeding material estimation. Biology, Breeding and Growing of Sunflower, Moscow, 77-80.

Soldatov, K.I., 1976 Chemical mutagenesis in sunflower breeding. In: Proc. $7^{\text {th }}$ Int. Sunflower Conf., Krasnodar, USSR, International Sunflower Association, Vlaardingen, Netherlands: 352-357.

\section{PRUEBA DE VINCULACIÓN DEL GEN IMR CON MUTACIONES ol, tph1 Y tph2 EN GIRASOL}

\author{
RESUMEN
}

El objetivo de esta investigación ha sido investigar la posibilidad de que exista un vínculo entre la resistencia hacia imidazolinona y las mutaciones en calidad del aceite en girasol. HA425 (ImrImr), la línea resistente hacia imidazolinona, pero con el normal contenido de ácidos grasos y tocoferol, fue utilizada como línea materna, tras la esterilización manual. La VK876, línea sensible hacia imidazolinona, y con el alto contenido del ácido oleico y cambiado contenido de tocoferol (OlOl tph1tph1 tph2tph2) fue utilizada como línea paterna. La prueba de vinculación, realizada en las generaciones $\mathrm{F}_{2}$ y $\mathrm{F}_{3}$, demostró que el gen $I m r$, que condiciona la resistencia hacia imidazolinona, hereda, independientemente de la mutación con el alto contenido del ácido oleico $\mathrm{Ol}$, la mutación con el alto contenido de $\beta$-tocoferol tph1 y la mutación con alto contenido de $\gamma$-tocoferol tph2. Fueron elegidos los genotipos recombinantes para la formación de las líneas consanguíneas. 


\title{
TEST DE LIAISON DU GÈNE IMR AVEC LES MUTATIONS ol, tph1 ET tph2 CHEZ LE TOURNESOL
}

\author{
RÉSUMÉ
}

Le but de cette recherche était d'observer la possibilité de l'existence d'un lien entre la tolérance envers l'imidazolinone et les mutations dans la qualité de l'huile chez le tournesol. Une lignée résistante d'imidazolinone HA425 (ImrImr) avec un contenu normal d'acides gras et de tocophérol a été utilisée comme lignée mère après stérilisation manuelle. Une lignée autogame VK876 avec contenu élevé d'acide oléique et composition de tocophérol altérée $(\mathrm{OlOl}$ tph1tph1 tph2tph2) et sensible à l'imidazolinone a servi de lignée mâle. Le test de liaison dans les générations $\mathrm{F}_{2}$ et $\mathrm{F}_{3}$ a montré que le gène $\mathrm{Imr}$ qui conditionne la résistance envers l'imidazolinone est hérité indépendamment de la mutation avec contenu élevé d'acide oléique $O l$, de la mutation avec contenu élevé de â-tocophérol tph1 et de la mutation avec contenu élevé de ã-tocophérol tph2. Des génotypes recombinants ont été choisis pour le développement de lignées autogames. 